\title{
Rituximab-induced hypersensitivity pneumonitis in a kidney transplant patient
}

\author{
Natália Silva ${ }^{1, *}$, Catarina Isabel Ribeiro ${ }^{1, *}$, Manuela Almeida ${ }^{3}$, Jorge Malheiro ${ }^{3}$, Sofia Pedroso ${ }^{3}$, Maria La Salete Martins ${ }^{3}$, Leonídio Dias ${ }^{3}$, António Castro \\ Henriques $^{3}$, António Cabrita ${ }^{3}$
}

* Both authors contributed equally to this work

1 Serviço de Nefrologia - Centro Hospitalar de Trás-os-Montes e Alto Douro

2 Serviço de Nefrologia - Centro Hospitalar de Vila Nova de Gaia/ Espinho

${ }^{3}$ Serviço de Nefrologia/ Unidade de Transplantação Renal - Centro Hospitalar Universitário do Porto, Porto - Portugal

\section{ABSTRACT}

Rituximab (RTX) is a chimeric anti-CD20 monoclonal antibody that has been associated with delayed pulmonary toxicity. In patients receiving RTX, hypersensitivity pneumonitis, although rare, should be considered in the appropriate clinical and radiographic setting. The authors present a case of hypersensitivity pneumonitis associated with a single dose of RTX treatment in a kidney transplanted patient with acute antibody-mediated rejection.

Key words: Drug-induced lung disease, hypersensitivity pneumonitis, kidney transplant, rituximab.

\section{INTRODUCTION}

Rituximab (RTX) is a chimeric anti-CD20 monoclonal antibody commonly used as treatment of different malignancies and autoimmune diseases. Kidney transplant is a new area for use of RTX, which is used to treat acute antibody-mediated rejection (ABMR) or as an immunomodulatory agent in $\mathrm{ABO}$ or human leukocyte antigen-incompatible grafts.

Side effects of RTX are frequently seen during the first infusion in up to $40 \%$ of patients and include fever, chills, nausea and rigors. ${ }^{1}$ Most of them are from studies in which RTX was given concomitantly with chemotherapeutic agents. ${ }^{2,3}$

Adverse respiratory reactions have been reported in up to $40 \%$ of patients in clinical trials, usually during initial administration, including cough, rhinitis, bronchospasm, dyspnea and sinusitis. ${ }^{1}$ More severe and delayed respiratory injuries such as interstitial pneumonitis, organized pneumoniae and diffuse alveolar hemorrhage are very rare. ${ }^{1,4}$

Hypersensitivity pneumonitis is a complex syndrome characterized by non-immunoglobulin E-mediated inflammation of lung parenchyma in response to an antigen, usually an inhaled allergen. Hypersensitivity pneumonitis can sometimes be a manifestation of drug-induced lung disease where clinical and medical imaging features are indistinguishable from those caused by inhaled organic antigens. ${ }^{4,5}$ RTX-induced interstitial lung disease is a rare but known complication. ${ }^{6,7}$ The authors present a case of hypersensitivity pneumonitis associated with RTX treatment in a kidney transplanted patient with acute ABMR.

\section{CASE REPORT}

A 19-year-old Caucasian woman with end-stage renal disease due to chronic pyelonephritis was kidney transplanted at the age of 9-years-old. In the context of multiple urinary tract infections, her renal function gradually aggravated to a basal serum creatinine of $3.00 \mathrm{mg} / \mathrm{dL}$. She was medicated with tacrolimus $4.5 \mathrm{mg} /$ day and prednisolone $5.0 \mathrm{mg} /$ day.

This patient was admitted at the transplantation unit with a recurrence of acute pyelonephritis and acute allograft dysfunction. At this time, she presented white blood cell count of $18.000 / \mathrm{uL}, \mathrm{C}$-reactive protein $113.0 \mathrm{mg} / \mathrm{L}$, serum creatinine $3.80 \mathrm{mg} / \mathrm{dL}$, urea $102 \mathrm{mg} / \mathrm{dL}$ and normal serum electrolytes. Although the inflammatory parameters decreased after initiating endovenous antibiotherapy, allograft function showed no improvement. An allograft biopsy was performed and histology findings were suggestive of acute cellular (Banf IA) and ABMR. Donor specific antibodies were identified, and she was successfully treated with plasmapheresis, intravenous immunoglobulin and one dose of RTX (500 mg). The treatment was considered successful due to gradual return to basal creatinine levels. At discharge, the prescribed immunosuppression was tacrolimus $4.5 \mathrm{mg} /$ day and prednisolone $5 \mathrm{mg} /$ day.

She was readmitted in our hospital one month later with asthenia, progressive dyspnea, dry cough and retrosternal pain of pleuritic characteristics with one week of evolution. She denied fever, weight loss, hemoptysis, abdominal pain, arthralgias, or other clinical manifestations.

At admission, physical examination was unremarkable except for bilateral inspiratory crackles. Her vital signs were as following: temperature 


\section{Figure 1}

Chest X-ray with no significant alterations

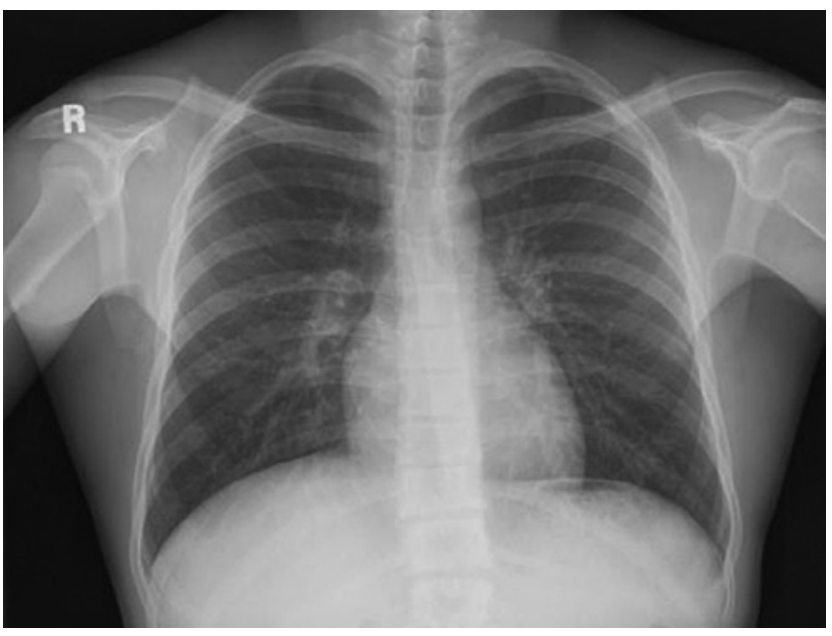

$36.0^{\circ} \mathrm{C}$, heart rate 78 beats/ min, blood pressure $94 / 48 \mathrm{mmHg}$, respiratory rate $19 / \mathrm{min}$ and oxygen saturation $93 \%$ on room air.

Laboratory analysis revealed the following values: hemoglobin 10.8 $\mathrm{g} / \mathrm{dL}$, white blood cell count of 27.000/uL (neutrophils $23.000 / \mathrm{uL}$; eosinophils $390 / \mathrm{uL}$ ), platelet count $160.000 / \mathrm{uL}$, serum creatinine $5.80 \mathrm{mg} / \mathrm{dL}$, urea $180 \mathrm{mg} / \mathrm{dL}, \mathrm{C}$-reactive protein $76.0 \mathrm{mg} / \mathrm{L}$ and normal serum electrolytes. Arterial blood gas presented pH 7.210, paO2 68.0 $\mathrm{mmHg}$, paCO2 $18.0 \mathrm{mmHg}$, bicarbonate $11.7 \mathrm{mmol} / \mathrm{L}$, lactate 1.0 $\mathrm{mmol} / \mathrm{L}$. Chest X-ray (Figure 1) and renal echography showed no significant alterations. Although she denied urinary symptoms, she had an uroculture positive for Morganella morganii.

The patient was initially admitted with the diagnosis of urinary sepsis and was treated with piperacillin-tazobactam. At the first day, the patient maintained hypotensive profile $(80 \mathrm{mmHg})$ with worsening of respiratory symptoms. A chest computed tomography (CT) revealed diffuse bilateral ground-glass opacities and multiple mediastinal ganglion formations (Figure 2). The patient underwent bronchoscopy with bronchoalveolar lavage. Bronchoalveolar lavage fluid (BAL) showed 40 red blood cells/ $\mathrm{mm}^{3}$ and 15 white blood cells/ $\mathrm{mm}^{3}$ (30\% monocytes, $50 \%$ lymphocytes and $20 \%$ polymorphonuclear cells). Special stain for cytomegalovirus and pneumocystis were negative. BAL fluid culture was negative for bacteria, fungi, and mycobacteria. Cytomegalovirus and BK virus blood viral load were negative. On the next day, the patient developed severe respiratory distress and was transferred to the Intensive Care Unit where she initiated non-invasive respiratory support.

BAL was obtained after 2 days of antibiotherapy. This could have masked a bacterial pneumonia; however, the rapid deterioration of symptoms under broad-spectrum antibiotics, the resulting of BAL tests and the medical imaging findings favored a hypersensitivity pneumonitis.

After discussion with a multidisciplinary team, the diagnosis of hypersensitivity pneumonitis was assumed. A meticulous review of

\section{Figure 2}

Computed tomography of the chest with diffuse bilateral ground-glass opacities and multiple mediastinal ganglion formations

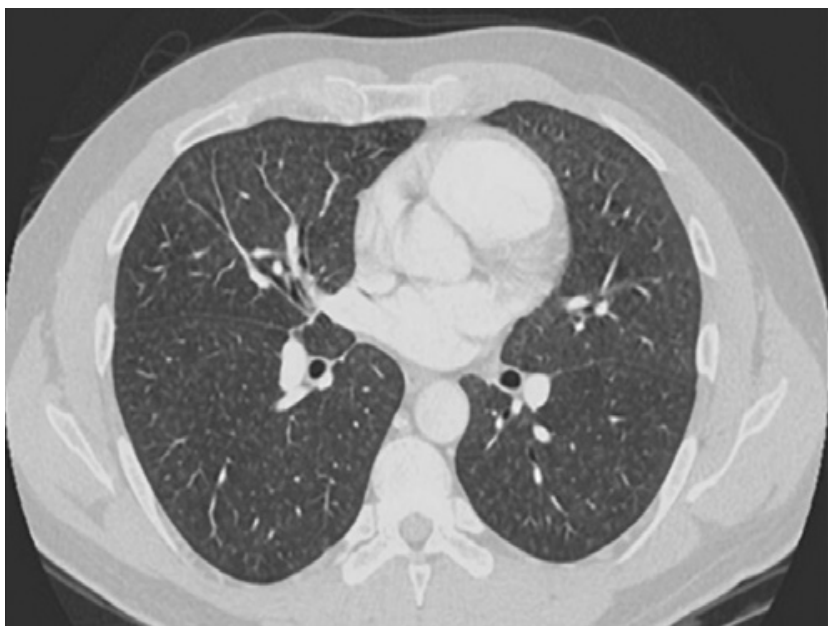

the patient's environmental and occupational exposure showed no obvious external cause. The maintenance dose of prednisolone that the patient was taking for her kidney transplant was adjusted to 1.0 $\mathrm{mg} / \mathrm{Kg} /$ day $(60 \mathrm{mg} /$ day) with an excellent clinical response. Within four days, her oxygenation improved sufficiently to permit a return to the nephrology ward with a resting room air pulse of oximetry saturation of $98 \%$ and no desaturation with ambulation. After two days she recovered kidney function to her basal and was discharged. The patient was then closely monitored in the kidney transplant consultation where she gradually tapered steroids.

Six months after discharge, she repeated the chest $\mathrm{CT}$, and it showed complete resolution of parenchymal findings. Pulmonary function tests were also performed and showed only slight reduction in diffusion capacity for carbon monoxide and normal rates of flow and gas exchange.

However, the patient presented progressive decline of renal allograft function and, at the current time, she is on peritoneal dialysis and under evaluation for a second renal transplant.

\section{DISCUSSION}

Hypersensitivity pneumonitis, also known as extrinsic allergic alveolitis, represents a group of pulmonary disorders mediated by an inflammatory reaction to inhalation of an allergen that can lead to lung fibrosis. ${ }^{8}$ So far more than 200 different antigens have been associated with the development of hypersensitivity pneumonitis, including plant products, animal products, aerosolized microorganisms, and organic chemicals. ${ }^{9}$ Although RTX is rarely linked to the development of hypersensitivity pneumonitis, in the last decade some reports have been described. ${ }^{7}$

RTX-induced hypersensitivity pneumonitis can affect patients of all ages and all racial backgrounds, with a similar incidence in both 
males and females. ${ }^{9}$ The time of onset, from the last TX infusion until symptom development or relevant abnormal radiology change, differ from 0 to 158 days, with a mean of 30 days. ${ }^{10}$

In the present case, and unlike other reports, the authors present a case of RTX-induced pneumonitis hypersensitivity in a young kidney transplanted patient one month after receiving a single dose of RTX. To the author's knowledge, there is no other similar case described in the literature.

Acute hypersensitivity pneumonitis is a pathology that leads to a typically clinical presentation, and symptoms may include fevers, rigors, myalgia, coughing, chest tightness, dyspnea, and leukocytosis. ${ }^{11}$ In the present case, asthenia, progressive dyspnea, dry cough and retrosternal pain of pleuritic characteristics were the symptoms presented. This patient also had an important elevation of inflammatory biomarkers, with blood cell count of $27.000 / u L$ (with 23.000/uL neutrophils) and C-reactive protein $76.0 \mathrm{mg} / \mathrm{L}$.

In this lung disease, chest radiography and high-resolution computed tomography of the chest can provide supportive diagnosis evidence. However, radiographic findings will vary according to the stage of the disease. ${ }^{8,11}$ In this patient, while chest radiography showed no significant alterations, computed tomography revealed diffuse bilateral ground-glass opacities and multiple mediastinal ganglion formations.

The pathogenic mechanism has yet to be elucidated. Due to the long half-life of RTX, prolonged B-cell depletion may interfere with lymphocyte crosstalk, causing cytotoxic T lymphocyte dysregulation, thereby promoting lung damage. ${ }^{2,12}$ Leukostasis in the pulmonary circulation and release of inflammatory cytokines have also been described as possible mechanisms. ${ }^{3,13}$

After discussion with a multidisciplinary team, the diagnosis of hypersensitivity pneumonitis was assumed. The suggestive clinical evaluation and the excellent corticosteroid response were important facts that supported this diagnosis. Transbronchial biopsy was not performed, and this could have provided relevant information. The typical histological features are interstitial lymphocytic infiltrates, cellular bronchiolitis and poorly formed (loose) non-necrotizing granulomas. ${ }^{14}$

It is unclear why our patient developed hypersensitivity pneumonitis while on low-dose prednisone. It is possible that low-dose prednisone was inadequate to suppress the development of pneumonitis. ${ }^{15}$

Patients who worsen despite corticosteroids have a poor outcome. In two systematic reviews, of all reported cases of RTX-induced interstitial lung disease, the mortality rate varied between 15 to $37 \%$. ${ }^{10,16}$
The authors consider that hypersensitivity pneumonitis is a disease in which a high degree of clinical suspension is required, and they recommend emphasizing the importance of early identification of worsening pulmonary symptoms in patients receiving RTX.

\section{CONCLUSION}

In patients receiving RTX, hypersensitivity pneumonitis, although rare, should be considered in the appropriate clinical and radiographic setting. The authors confirmed that a complete and rapid resolution is possible after treatment with steroids.

Disclosure of potential conflicts of interest: none declared

\section{References}

1. Genentech. Rituxan: highlights of prescribing information, revised December 2019. https://www. gene.com/

2. Alexandrescu DT, Dutcher JP, O'Boyle K, Albulak M, Oiseth S, Wiernik PH. Fatal intra-alveolar hemorrhage after RTX in a patient with non-Hodgkin lymphoma. Leuk Lymphoma 2004; 45: 2321-2325 .

3. Burton C, Kaczmarski R, Jan-Mohamed R. Interstitial pneumonitis related to RTX therapy. N Engl J Med 2003; 348: 2690-2691.

4. Kimby E. Tolerability and safety of RTX. Cancer Treat Rev 2005; 31:456-473.

5. Patel AM, Ryu JH, Reed CE. Hypersensitivity pneumonitis: current concepts and future questions. J Allergy Clin Immunol 2001; 108: 661-670.

6. Tonelli AR, Lottenberg R, Allan RW, Sriram PS. RTX-induced hyper-sensitivity pneumonitis. Respiration 2009; 78: 225-229.

7. Heresi GA, Farver CF, Stoller JK. Interstitial pneumonitis and alveolar hemorrhage complicating use of RTX. Respiration 2008; 76: 449-453.

8. Pereira C, Gimenez A, Kuranishi L, Storrer K. Chronic hypersensitivity pneumonitis. J Asthma Allergy 2016; 9: 171-181.

9. Nogueira R, Melo N, Novais e Bastos H, Martins N, Delgado L, Morais A, et al. Hypersensitivity pneumonitis: antigen diversity and disease implications. Pulmonol 2019; 25(2): 97-108.

10. Hadjinicolaou AV, Nisar MK. Non-infectious pulmonary toxicity of RTX: a systematic review. Rheumatology. 2012; 51(4): 653-662.

11. D'souza RS, Donato A. Hypersensitivity pneumonitis: an overlooked cause of cough and dyspnea. J Community Hosp Intern Med Perspect 2017; 7(2): 95-99.

12. Selenko N, Maidic O. CD20 antibody (C2B8)-induced apoptosis of lymphoma cells promotes phagocytosis by dendritic cells and cross-priming of CD8+ cytotoxic T cells. Leukemia, 2001; 15 : 1619-1626.

13. Bienvenu J, Chvetzoff R. Tumor necrosis factor alpha release is a major biological event associated with RTX treatment. Hematol J 2001; 2: 378-384.

14. Silva $\mathrm{Cl}$, Churg A, Müller NL. Hypersensitivity pneumonitis: spectrum of high-resolution $\mathrm{CT}$ and pathologic findings. AJR Am J Roentgenol 2007; 188: 334-344.

15. Swords R, Power D, Fay M, O'Donnell R, Murphy PT. Interstitial pneumonitis following RTX therapy for immune thrombocytopenic purpura (ITP) Am J Hematol 2004; 77: 103-104.

16. Wagner SA, Mehta AC, Laber DA. RTX-induced interstitial lung disease. Am J Hematol 2007; 82: 916-919.

\section{Correspondence to:}

Natália Silva, MD

Centro Hospitalar de Trás-Os-Montes e Alto Douro - Unidade de Vila Real

Avenida Noruega, s/n | 5000-508 Lordelo, Vila Real - Portugal

E-mail: nataliasofia_1@hotmail.com

https://orcid.org/0000-0002-7206-3276 